\title{
Efecto de la suplementación con selenio orgánico sobre la calidad de la canal y la vida útil de la carne de vaquillonas terminadas en feedlot
}

\section{Effect of organic selenium supplementation on carcass quality and meat shelf life in feedlot finished heifers}

\author{
Juan Franco ${ }^{1^{*}} 0000-0003-1198-8213$ \\ Álvaro Simeone ${ }^{1}$ 0000-0001-5240-5710 \\ Virginia Beretta ${ }^{1}$ 0000-0002-4762-5185 \\ Carolina Realini ${ }^{2}$ 0000-0003-2036-316X \\ Ali Saadoun ${ }^{3}$ 0000-0003-2251-6748 \\ Alejandra Terevinto ${ }^{1}$ 0000-0003-3737-7859 \\ ${ }^{1}$ Departamento de Producción Animal y Pasturas. Facultad de Agrono- \\ mía EEMAC. *Autor para correspondencia: jufrafagro@gmail.com \\ ${ }^{2}$ Polo de Producción y Reproducción de Rumiantes. Cenur Litoral Norte. \\ ${ }^{3}$ Departamento de Fisiología y Nutrición. Facultad de Ciencias. \\ Veterinaria.
}
Cristina Cabrera ${ }^{1}$ 0000-0002-7964-6669
Agustin Costanzo ${ }^{7}$ 0000-0002-32209613
Gonzalo Ravecca ${ }^{7}$ 0000-0001-8561-0622
Antonella Goyeneche ${ }^{4}$ 0000-0002-6728-2765
Rafael Delpiazzo ${ }^{5}$ 0000-0003-3461-0395
Oscar Bentancur ${ }^{6}$ 0000-0003-0509-5793
${ }^{4}$ Departamento de Tecnología de los Alimentos. Cenur Litoral Norte.
${ }^{5}$ Departamento de Salud en los Sistemas Pecuarios. Facultad de Veterinaria.
${ }^{6}$ Departamento de Estadística y Cómputos. Facultad de Agronomía EE- MAC.
${ }^{7}$ Estudiantes en Tesis. Facultad de Agronomía EEMAC

Veterinaria (Montevideo) Volumen 56

№ 214 (2020 Jul - Dic) e20205621405

\section{Resumen}

El objetivo del presente trabajo fue evaluar el efecto de la suplementación con selenio orgánico (Selplex ${ }^{\circledR}$ ) sobre la calidad de la canal y la vida útil de la carne de vaquillonas en régimen de estabulación. Se utilizaron 30 vaquillonas de la raza Hereford: 14 de 20 meses de edad, con un peso inicial de $323 \pm 26,3$ $\mathrm{kg}$, y 16 de 36 meses y $405 \pm 30,5 \mathrm{~kg}$. Los animales fueron estabulados en corrales individuales y sorteados dentro de cada categoría de edad a dos tratamientos: Control y Suplementación con selenio orgánico a razón de $0.9 \mathrm{mg} \mathrm{Se} / \mathrm{kg} \mathrm{MS} /$ día. Todos los animales fueron alimentados con una ración totalmente mezclada (RTM) incluyendo $15 \%$ de fardo de moha (Setaria itálica) y $85 \%$ de ración comercial para engorde de vacunos. Los animales fueron faenados luego de 62 días de alimentación. Las determinaciones de evolución de color, oxidación de lípidos y proteínas se realizaron sobre muestras envasadas, usando un film permeable al oxígeno y almacenadas durante 12 días en vitrina refrigerada. La suplementación con Se resultó en un aumento de los niveles sanguíneos de glutatión peroxidasa (GSH-Px) (195.9 vs. 259.7 U/gr Hg, p<0.0001), en una disminución de la oxidación lipídica (0.65 vs. $0.57 \mathrm{mg}$ de malondialdehido $/ \mathrm{kg})(\mathrm{p}<0.05)$ y de la fuerza de corte después de 7 días de maduración (3.22 vs. $2.68 \mathrm{~kg})(\mathrm{p}<0.05)$. Las vaquillonas suplementadas de 20 meses obtuvieron niveles sanguíneos más altos de GSH-Px (242vs. $213 \mathrm{U} / \mathrm{gr} \mathrm{Hg})(\mathrm{p}<0.05)$ y se detectó un menor sabor rancio en relación a las de 36 meses ( 2.9 vs. 3.6) $(\mathrm{p}<0.05)$. La suplementación con selenio orgánico puede ser un alternativa a utilizar para disminuir la oxidación lipídica, sin embargo no tuvo efecto en la evolución del color y en reducir la oxidación de proteínas de la carne (0.31 vs.0.35 nmoles $/ \mathrm{mg})(\mathrm{p} \geq 0.05)$.

Palabras clave: Bovinos, Selenio orgánico, Vida útil, Calidad de carne, Feedlot.
Recibido: 05/02/2020

Aceptado: 12/10/2020

The objective of this work was to evaluate the effect of organic selenium supplementation (Selplex ${ }^{\circledR}$ ) on heifer's carcass quality and meat shelf life in a feedlot system. Thirty Hereford heifers were used: 14 of 20 months of age, with an initial weight of 323 $\pm 26.27 \mathrm{~kg}$, and 16 of 36 months and $405 \pm 30.52 \mathrm{~kg}$. Animals were housed in individual pens and drawn within each age category to 2 treatments: Control and Supplementation with organic selenium at a rate of $0.9 \mathrm{mg} \mathrm{Se} / \mathrm{kgDM} /$ day. All animals were fed a fully mixed ration (RTM) including 15\% moha bale (Setaria itálica) and $85 \%$ commercial ration for fattening cattle. Animals were slaughtered after 62 days of feeding. Color evolution, lipid and protein oxidation were measure on sample packed using an oxygen permeable film and stored during 12 days in a refrigerated display case. Selenium supplementation resulted in an increase level of glutathione peroxidase (GSH-Px) (195.9 vs 259.7 $\mathrm{U} / \mathrm{gr} \mathrm{Hg}, \mathrm{p}<0.0001)$, a decrease lipid oxidation ( $0.65 \mathrm{vs} .0 .57 \mathrm{mg}$ $\mathrm{MDA} / \mathrm{kg})(\mathrm{p}<0.05)$ and shear force after 7 days of ageing $(3.22$ vs. $2.68 \mathrm{~kg})(\mathrm{p}<0.05)$. Twenty months heifers supplemented with selenium achieved higher levels of GSH-Px (242 vs. $213 \mathrm{U} / \mathrm{gr}$ $\mathrm{Hg})(\mathrm{p}<0.05)$ and was detected a lower rancid flavor in relation to those of 36 months ( 2.9 vs. 3.6) $(\mathrm{p}<0.05)$. Organic selenium supplementation may be an alternative to decrease meat lipid oxidation, however it had no effect on color evolution and in reducing protein oxidation ( 0.31 vs. 0.35 nmoles $/ \mathrm{mg})(\mathrm{p} \geq 0.05)$.

Keywords: Beef cattle, Organic Selenium, Shelf life, Meat quality, Feedlot. 


\section{Introducción}

La mayor parte de la literatura atribuye a la carne proveniente de sistemas de engorde a corral una mayor terneza, un mayor veteado y un color más blanco de la grasa de cobertura respecto a la procedente de sistemas pastoriles. Estas características están particularmente influenciadas por la tasa de descenso y el pH final que alcance la carne, explicada por la mayor concentración de glucógeno muscular (Immonem et al., 2000; O'Sullivan et al., 2003) y un enfriado más lento en canales con mayor nivel de engrasamiento. Este menor $\mathrm{pH}$ en canales provenientes de sistemas en confinamiento está altamente correlacionado con el color, principalmente con la luminosidad $\left(\mathrm{L}^{*}\right)$, generando carnes más luminosas (Klont et al., 1999).

La menor edad de faena, una mayor deposición de colágeno soluble, y un mayor veteado determinan una mayor terneza y jugosidad de la carne de animales en confinamiento. La faena de animales a una menor edad, y la ausencia de pigmentos provenientes de la pastura como el beta caroteno, dan como resultado una grasa de cobertura más blanca comparada con la tonalidad amarillenta de la grasa, propia de la alimentación pastoril (Realini et al., 2004).

Sin embargo, es importante remarcar que la concentración de antioxidantes presentes en la carne también tiene importancia sobre el color, sabor y vida útil, ya que protegen las membranas de las fibras musculares impidiendo la peroxidación de los lípidos durante el almacenamiento. Estos antioxidantes disminuyen con la utilización de granos en la dieta, debido a la menor concentración de Vitamina E en relación a los forrajes, produciendo una disminución de la estabilidad de los lípidos de la carne y acortando la vida útil (Descalzo y Sancho, 2008; Realini et al., 2004).

En el caso de la carne vacuna fresca está documentado que la carga microbiana y la temperatura de refrigeración, son especialmente preponderantes en determinar su vida útil, dadas sus condiciones óptimas en nutrientes y las pocas barreras naturales que las mismas poseen para el desarrollo de una gran variedad de microorganismos. Sin embargo, el control estricto de ambos parámetros (carga microbiana y temperatura) solo es suficiente para alcanzar un período limitado de comercialización. La oxidación de los lípidos es una de las principales causas del deterioro de la calidad de la carne, afectando el color, sabor, textura y su valor nutricional (Masana et al., 2002).

La decoloración de la carne es una función combinada de oxidación del pigmento muscular (oximioglobina a metamioglobina) y oxidación lipídica que ocurre en la grasa intramuscular, grasa intermuscular y/o fosfolípidos de la membrana. Prolongar el tiempo que la carne conserva su apariencia de color rojo cereza brillante se puede lograr mediante la prevención o el retraso de la oxidación del pigmento minimizando la formación de metamioglobina (Geay et al., 2001).
En el tejido muscular, las funciones antioxidantes del selenio y vitamina E persisten después del sacrificio y retrasan la aparición de reacciones de oxidación en la carne y los productos cárnicos. Si bien existe información sobre el efecto de la suplementación de selenio sobre la calidad de la carne, la mayoría de los trabajos se han realizado en aves (Downs et al., 2000; Edens, 1996; Naylor et al., 2000) y cerdos (Mahan y Parret, 1996) en comparación con la carne bovina. A su vez, no hay antecedentes reportados en la literatura en relación al efecto del selenio en la oxidación proteica.

Los suplementos de selenio se comercializan bajo dos formas: las sales minerales inorgánicas, tales como el selenito de sodio o el selenato; o formas orgánicas, en la que la selenometionina es la forma predominante. La selenometionina se retiene en las proteínas tisulares en mayor medida que selenocisteína y las formas inorgánicas de selenio.

El alto costo de la vitamina E, los mayores niveles de engrasamiento alcanzados en regímenes de estabulación, la importancia del Selenio como antioxidante y nutriente esencial para la salud animal y humana, hacen relevante estudiar el efecto de la suplementación con Selenio orgánico.

El objetivo de este trabajo fue evaluar el efecto de la suplementación con selenio orgánico (Selplex ${ }^{\circledR}$ ) sobre la calidad de la canal y la vida útil de la carne de vaquillonas en régimen de estabulación.

\section{Materiales y métodos}

Se utilizaron 30 vaquillonas de la raza Hereford: 14 de 20 meses de edad, con un peso inicial de $323 \pm 26,3 \mathrm{~kg}$, y 16 de 36 meses y $405 \pm 30,5 \mathrm{~kg}$, provenientes del rodeo experimental de la EEMAC. Luego de un período de acostumbramiento inicial de 18 días, el período experimental de confinamiento tuvo una duración de 62 días. Los animales fueron estabulados en corrales individuales a cielo abierto $\left(25,2 \mathrm{~m}^{2}\right.$ /animal) y dentro de cada categoría fueron asignados al azar a uno de dos tratamientos: 1) Control, sin suplementación ( $\mathrm{n}=15)$; y 2) Selenio; suplementación con la forma orgánica de levadura de selenio $(n=15)$ (Sel -Plex 2000, Alltech ${ }^{\circledR}$ ) a razón de $0.9 \mathrm{mg} \mathrm{Se} / \mathrm{kg} \mathrm{MS/día.}$

El alimento fue ofrecido ad libitum. Esto se logró en base a una lectura diaria de comedero con el objetivo de que hubiera siempre un excedente de alimento del día anterior $(5 \%$ de lo suministrado). La dieta fue distribuida en tres comidas diarias, ofreciéndose en la mañana (8:00 hs) el 20\% de la misma junto con la suplementación de Selplex ${ }^{\circledR}$ en los animales que tenían ese tratamiento, asegurando la ingesta completa del suplemento. El 80\% restante de la dieta fue suministrado en partes iguales en el medio día (13:00 hs) y tarde (17:00 hs).

Los animales contaban con agua a voluntad la cual se reponía diariamente y se realizaba limpieza de bebederos semanalmente 
asegurando la calidad de la misma y evitando posibles efectos negativos sobre la performance animal.

Todos los animales fueron alimentados con control diario de consumo, con una ración totalmente mezclada (RTM) incluyendo $15 \%$ de fardo de moha (Setaria itálica) y $85 \%$ de ración comercial para engorde de vacunos. Los componentes del concentrado fueron los siguientes: sorgo, maíz, cebada, avena, afrechillo de arroz, afrechillo de trigo, harina de soja, torta de soja extrusada, torta de soja (expeller), harina de lino, harina de girasol, raicilla, brotes de cebada, pellets de citrus (pulpa), melaza, carbonato de calcio y fosfato bicálcico. En la tabla 1 se muestra la composición química de la ración utilizada.

A inicio y fin del período de alimentación a corral, se tomaron muestras de sangre a cada animal, para la posterior determinación de la glutatión peroxidasa como indicador del nivel de selenio (Se) en sangre. La actividad sanguínea de glutatión peroxidasa (GSH-Px) se analizó mediante una técnica cinética compuesta NADPH-dependiente (Paglia y Valentine, 1967).

Las vaquillonas fueron faenadas en un frigorífico comercial después de 62 días de engorde a corral. El PV a faena se determinó individualmente en la planta frigorífica luego de 24 horas de ayuno. Al finalizar el proceso de faena se obtuvo el peso de canal caliente (PCC). El rendimiento de los animales se calculó como la relación del peso canal caliente y el peso vivo a la faena, expresado como porcentaje.

Las canales permanecieron en cámaras de frío durante 48 horas. El peso de canal fría fue registrado a la salida de la cámara de frío previo al cuarteado. A las 48 horas post-sacrificio se determinó:

- $\quad$ el área de ojo de bife (AOF) del músculo Longissimus dorsi, mediante el calco en papel acetato del contorno del mismo en la $10^{\mathrm{a}}$ costilla;

- $\quad$ el pH final mediante un peachímetro Hanna HI 99163 (Rumania, CE) con electrodo de penetración;

- $\quad$ el espesor de grasa subcutánea (EGS) en el centro del área de ojo de bife con regla milimetrada.

- $\quad$ La capacidad de retención de agua (CRA) por el método de compresión sobre la porción torácica del Longisssimus dorsi (Albertí et al., 2005).

Tabla 1: Composición química de la ración comercial.

\begin{tabular}{lr}
\hline Materia seca (\%) & 86,6 \\
Fibra cruda (\%) & 10 \\
Minerales totales (\%) & 10 \\
$\mathrm{Cl} \mathrm{Na}(\%)$ & 2 \\
Cenizas (\%) & 2 \\
Nitrógeno orgánico disuelto (ppm) & 5 \\
Proteína (\%) & 12 \\
Extracto etéreo (\%) & 2,5 \\
\hline
\end{tabular}

Se extrajeron las muestras para las evaluaciones de textura instrumental del músculo Longissimus dorsi entre la $10^{\mathrm{a}}$ costilla y $1^{\text {a }}$ vértebra lumbar de $2,5 \mathrm{~cm}$ de espesor, fueron envasadas al vacío y maduradas a temperatura de refrigeración durante 0 y 7 días. Luego fueron congeladas hasta su posterior análisis. Las determinaciones de la fuerza de corte se realizaron mediante cocción de las muestras en baño termostatizado a una temperatura interna de $70^{\circ} \mathrm{C}$. Las submuestras cilíndricas de $1,27 \mathrm{~cm}$ de diámetro fueron extraídas en dirección de las fibras y sometidas a la fuerza de corte de la cizalla Warner Bratzler, a una velocidad de $100 \mathrm{~mm}$ por minuto mediante un equipo Instron 3342 (USA) (Beltrán y Roncalés, 2000).

Para la determinación de color, las muestras de carne fueron envasadas individualmente en bandejas de poliespam usando un film permeable al oxígeno y se almacenaron a una temperatura de $2-4^{\circ} \mathrm{C}$ en una vitrina refrigerada. El color instrumental se determinó a los 0 (48 horas post mortem), 3, 6, 9 y 12 días de almacenamiento. Las coordenadas de color ( $L^{*}, a^{*}$ y b*) se determinaron por triplicado luego de una hora de exposición al oxígeno, mediante un colorímetro Konica Minolta CR-400 (Japón) con un diámetro de área de $8 \mathrm{~mm}$. Con los valores de $\mathrm{a}^{*}$ y de $b^{*}$ se calculó la cromaticidad o Croma: $C^{*}=\sqrt{ } a^{2}+b^{2}$ y el Tono $\mathrm{T}=\operatorname{tang}^{-1}\left(\mathrm{~b}^{*} / \mathrm{a}^{*}\right)$.

La oxidación de lípidos y de proteínas durante el almacenamiento de la carne se midió en los días 0, 6 y 12. En el caso de los lípidos se utilizó la técnica TBARS (determinación de sustancias reactivas al ácido tiobarbitúrico) representadas por malondialdehido (MDA), según el método de Lynch y Frei (1993). Se midió la absorbancia del sobrenadante en un espectrofotómetro (Genesys-6) a $535 \mathrm{~nm}$ de longitud de onda. Se calculó la concentración del MDA de las muestras utilizando su coeficiente de extinción molar $\left(156.000 \mathrm{M}^{-1} \mathrm{~cm}^{-1}\right)$ y los resultados se expresaron en $\mathrm{mg}$ de $\mathrm{MDA} / \mathrm{kg}$ músculo.

Para la determinación de la oxidación de proteínas se siguió el método descrito por Mercier et al., (2004). La absorbancia se midió en un espectrofotómetro (Genesys-6) a 370 nm de longitud de onda. Para la determinación del contenido total de carbonilos de las muestras se utilizó el método DNPH (dinitrofenilhidrazina). Se calculó utilizando su coeficiente de extinción molar $\left(22.000 \mathrm{M}^{-1} \mathrm{~cm}^{-1}\right)$ y los resultados se expresaron en nmoles de DNPH/mg proteína (Terevinto, 2010).

Para el análisis sensorial, el diseño utilizado fue incompleto (no todos los tratamientos fueron evaluados por el mismo panelista) y equilibrado (todos los tratamientos se analizaron la misma cantidad de veces). Este análisis fue realizado mediante 10 panelistas calificados previamente reclutados y entrenados los cuales evaluaron 8 muestras de carne, en 3 sesiones. El orden de degustación de las muestras de cada panelista fue aleatorio. Se evaluaron un total de 240 muestras correspondientes a los 2 tratamientos (Control y Selenio) y a cuatro niveles (vaquillonas de 20 y 36 meses; y 0 y 7 días de maduración). Las muestras fueron envueltas en papel aluminio y cocinadas en un grill de doble 

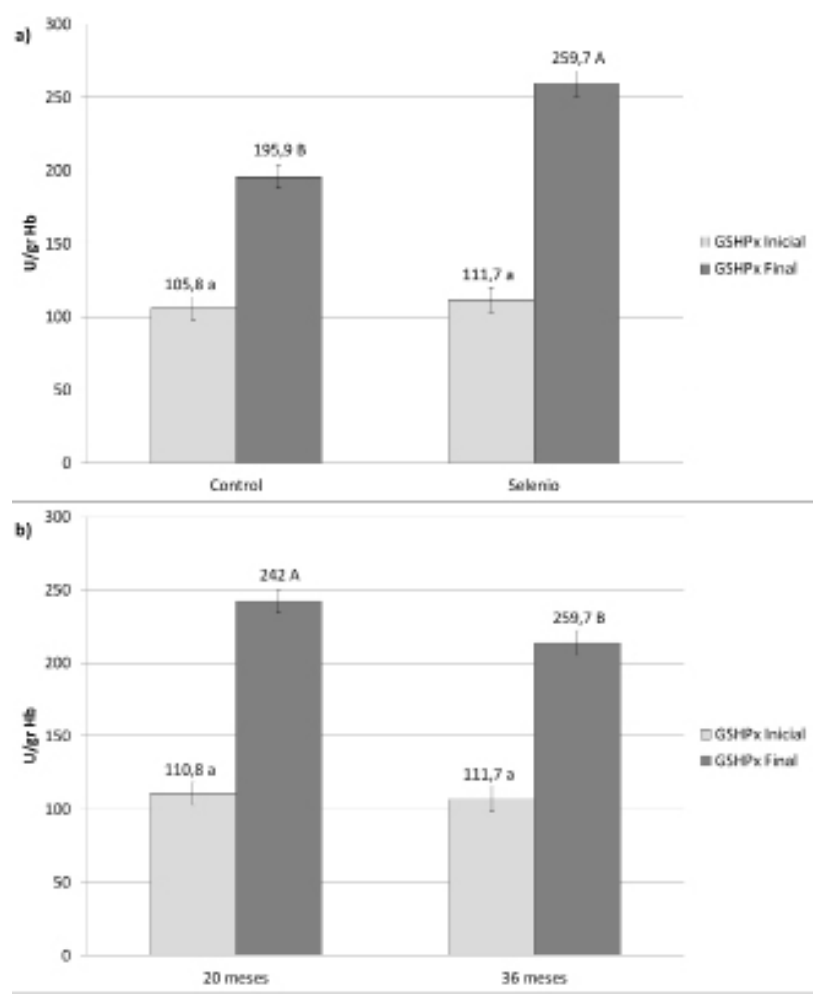

Figura 1: Niveles de la enzima glutatión peroxidasa (GSH -Px) en sangre según tratamientos (a) y edad de las vaquillonas (b) al inicio y al final del experimento. Letra minúscula (a) corresponden a valores de GSH-Px inicial. Letras mayúsculas (A y B) corresponden a valores de GSH-Px final del experimento.

plancha, donde una termocupla insertada en el centro de cada bife aseguraba una cocción uniforme a una temperatura de $70^{\circ} \mathrm{C}$. Luego de la cocción se subdividieron las muestras para utilizarlas luego en el panel, envolviéndolas nuevamente en papel aluminio, etiquetadas previamente con un número de tres cifras. Cada panelista contaba con una planilla para la correspondiente evaluación, la cual consistía en evaluar terneza, sabor rancio y sabor extraño, usando una escala estructurada del 1 al 9 en forma creciente, siendo 1 NADA y 9 MUY INTENSO.
Para el análisis estadístico de los niveles de glutatión peroxidasa en sangre, las variables de canal y calidad instrumental de la carne se utilizó un diseño de parcelas al azar con arreglo factorial de tratamientos, considerando al animal como unidad experimental.

Se realizó un análisis de varianza mediante el procedimiento MIXED del paquete estadístico SAS versión 9.1 (SAS Institute, Cary, NC, 2012), considerando que un efecto es estadísticamente significativo cuando la probabilidad de error Tipo I sea menor al $5 \%$.

Para analizar el efecto de los tratamientos, orden de degustación, sesión de degustación y jueces sobre los puntajes de terneza, sabor rancio y sabor extraño, se ajustó un modelo lineal generalizado asumiendo una distribución multinominal ordinal de la variable de respuesta. Se compararon los perfiles de probabilidades acumulativas de los distintos niveles de los factores estudiados mediante contrastes simples. Luego se estimaron las probabilidades de cada punto de la escala y se obtuvo una medida estimada del puntaje de degustación por medio de la función nexo logit acumulativa. Se usó el procedimiento Glimmix del paquete estadístico SAS versión 9.1 (SAS Institute, Cary, NC, 2012).

\section{Resultados}

Al analizar los datos no se identificaron efectos de interacción entre los tratamientos por lo que se presentarán los resultados de los efectos principales con la excepción de la fuerza de corte y del parámetro sensorial correspondiente al sabor rancio.

La suplementación con Se determinó un aumento de la actividad de GSH-Px en sangre $(p<0.001)$. Esta respuesta fue mayor en las vaquillonas de 20 meses en relación a las de 36 meses de edad $(\mathrm{p}=0.0157)$ (Figura 1).

Las características de la canal y de la carne no se vieron afectadas por efecto de los tratamientos pero sí por la edad de las vaquillonas (Tabla 2).

Tabla 2: Efecto del tratamiento con selenio (Sel-Plex, Alltech®) y la edad de las vaquillonas sobre las características de calidad de la canal y de la carne.

\begin{tabular}{lccccccc}
\hline & \multicolumn{9}{c}{ Tratamiento } & \multicolumn{5}{c}{ Edad vaquillonas } \\
\hline & Control & Selenio & P & 20 meses & 36 meses & P & RMSE \\
PV campo, kg & 445.75 & 458.93 & 0.2457 & 405.11 & 499.57 & $<.0001$ & 32.867 \\
PV faena, kg & 425.31 & 434.64 & 0.2224 & 387.31 & 472.64 & $<.0001$ & 37.483 \\
PCC, kg & 217.32 & 225.43 & 0.1547 & 201.94 & 240.80 & $<.0001$ & 18.544 \\
Rendimiento, \% & 51.92 & 51.98 & 0.7829 & 51.26 & 52.63 & 0.0470 & 1.749 \\
Merma (\%) & 1.3 & 1.3 & 0.9911 & 1.08 & 1.48 & 0.0960 & 1.08 \\
EGS, mm & 11.13 & 11.29 & 0.9199 & 10.34 & 12.07 & 0.5244 & 3.080 \\
AOB, cm & 49.03 & 52.23 & 0.2232 & 48.74 & 52.52 & 0.5888 & 6.568 \\
pH & 5.58 & 5.65 & 0.2653 & 5.67 & 5.57 & 0.1271 & 0.158 \\
CRA, \% & 30.51 & 28.32 & 0.3753 & 28.26 & 30.56 & 0.0811 & 6.858 \\
WB (Kg) & 3.05 & 2.76 & 0.4792 & 2.78 & 2.95 & 0.1275 & 0.123 \\
\hline
\end{tabular}

$\mathrm{PV}=$ peso vivo, $\mathrm{PCC}=$ peso canal caliente, $\mathrm{Merma}=\mathrm{PCC}-\mathrm{PC}$ fría $/ 100, \mathrm{EGS}=$ espesor de grasa subcutánea, $\mathrm{AOB}=$ área de ojo de bife del longissimus dorsi en la $10^{\circ}$ costilla, $\mathrm{CRA}=$ capacidad de retención de agua, $\mathrm{WB}=$ fuerza máxima de corte de la cizalla de Warner Bratzler. 


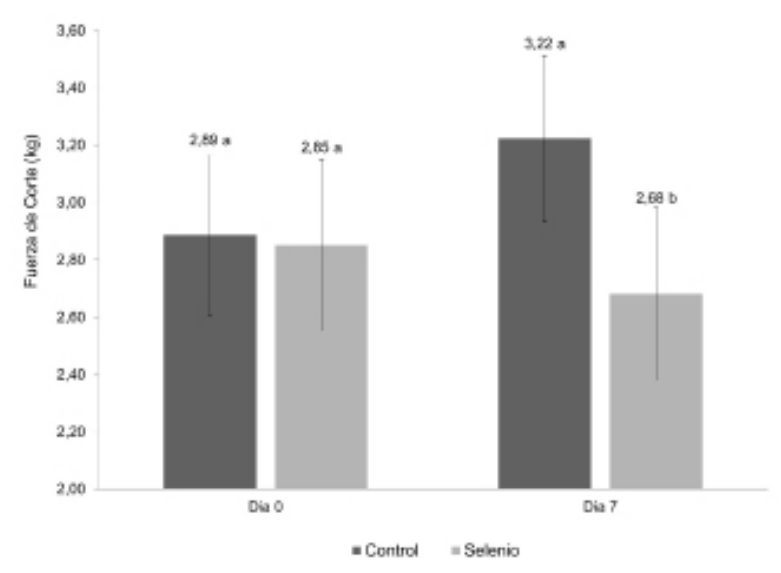

Figura 2: Valores de fuerza de corte en los días 0 y 7 de maduración postmortem según tratamientos.

Las vaquillonas control y suplementadas tuvieron una pérdida de peso entre el campo y frigorífico (desbaste) de un 4.6 y $5.2 \%$ respectivamente, con un rendimiento canal del $52 \%$, niveles de terminación correspondientes a animales terminados en confinamiento, y valores de fuerza de corte (WB) equivalentes a una carne tierna.

En relación a la edad, las vaquillonas de 36 meses presentaron mayores pesos de faena y mayores pesos de canal $(p<.0001)$, logrando así una superioridad de $1.3 \%$ en el rendimiento $(\mathrm{p}=$ 0.0470).

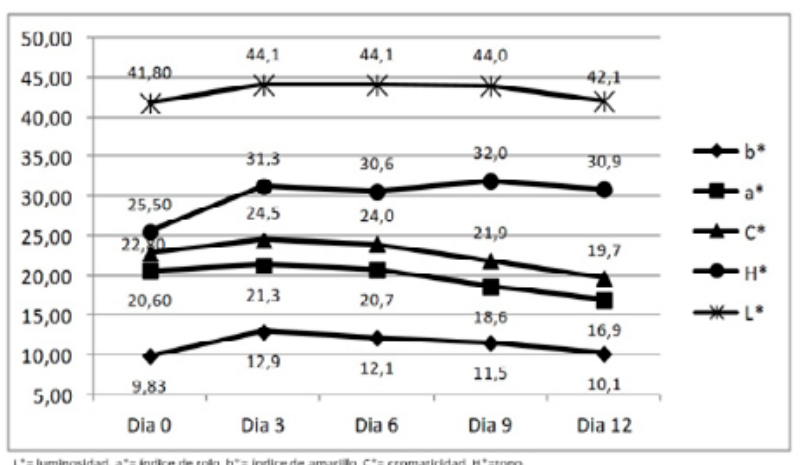

Figura 3: Evolución de los parámetros de color según días de exposición en vitrina refrigerada.

Si bien no se observaron diferencias en la fuerza de corte por efecto de los tratamientos o edad de las vaquillonas, se identificó una interacción entre la suplementación con Se y los días de maduración, dando como resultado una menor fuerza de corte para los animales suplementados con Se respecto a los controles después de 7 días de maduración (figura 2), alcanzando valores de 2.68 y $3.22 \mathrm{~kg}$ respectivamente $(\mathrm{p}=0.0495)$.

Tabla 3: Efecto del tratamiento con selenio (Sel-Plex, Alltech $\left.{ }^{\circledR}\right)$ sobre los niveles de Malondialdehido (MDA) y carbonilos (oxidación proteica).

\begin{tabular}{cccc}
\hline & Control & Selenio & P \\
\hline MDA (mg/kg) & 0.65 & 0.57 & 0.048 \\
Días de exposición & & & $<.0001$ \\
$\mathbf{0}$ & $0.52 \mathrm{~A} \mathrm{a}$ & $0.50 \mathrm{a}$ & \\
$\mathbf{6}$ & $0.61 \mathrm{~B} \mathrm{a}$ & $0.55 \mathrm{~b}$ & 0.390 \\
$\mathbf{1 2}$ & $0.70 \mathrm{C} \mathrm{a}$ & $0.65 \mathrm{~b}$ & 0.021 \\
& & & 0.35 \\
Carbonilos (nmoles/mg) & 0.31 & $0.28 \mathrm{a}$ & $0.34 \mathrm{a}$ \\
Días de exposición & & $0.43 \mathrm{a}$ & \\
$\mathbf{0}$ & $0.25 \mathrm{~A} \mathrm{a}$ & & \\
$\mathbf{1 2}$ & $0.33 \mathrm{~B} \mathrm{a}$ & $0.39 \mathrm{~B} \mathrm{a}$ & \\
\hline
\end{tabular}

Letras diferentes en la misma columna (A, B, C) difieren estadísticamente. Letras diferentes en la misma fila (a y b) difieren estadísticamente.

Tabla 4: Efecto de la suplementación con selenio y edad a la faena sobre los parámetros del color de la carne envasada con film permeable al oxígeno y en exposición en vitrina refrigerada durante 12 días.

\begin{tabular}{lccccc}
\hline \multicolumn{1}{c}{ Tratamiento } & $\mathbf{L}^{*}$ & $\mathbf{a}^{*}$ & $\mathbf{b}^{*}$ & $\mathbf{C}^{*}$ & $\mathbf{H}^{*}$ \\
\hline $\begin{array}{l}\text { Suplemento } \\
\text { Control }\end{array}$ & 43.67 & 19.70 & 11.47 & 22.84 & 30.34 \\
Selenio & 42.82 & 19.55 & 11.15 & 22.55 & 29.78 \\
$\mathbf{P}$ & 0.2554 & 0.2831 & 0.1834 & 0.1992 & 0.4603 \\
& & & & & \\
Edad animal & & & & & \\
$\mathbf{2 0}$ meses & 43.38 & 18.86 & 11.01 & 21.91 & 30.38 \\
$\mathbf{3 6}$ meses & 43.12 & 20.39 & 11.60 & 23.48 & 29.75 \\
$\mathbf{P}$ & 0.9999 & $<.0001$ & 0.0121 & $<.0001$ & 0.1316 \\
\hline $\mathrm{L}^{*}=$ luminosidad, $\mathrm{a}^{*}=$ índice de rojo, $\mathrm{b}^{*}=$ índice de amarillo, $\mathrm{C}^{*}=$ cromaticidad, $\mathrm{H}^{*}=$ tono.
\end{tabular}


Tabla 5: Atributos de la carne del panel sensorial según tratamiento, tiempo de maduración y edad del animal.

\begin{tabular}{|c|c|c|c|c|}
\hline Variables analizadas & & $\begin{array}{c}\text { Terneza } \\
(1-9)^{1}\end{array}$ & $\begin{array}{c}\text { Sabor Rancio } \\
(1-9)\end{array}$ & $\begin{array}{c}\text { Sabor extraño } \\
(1-9)\end{array}$ \\
\hline \multirow{3}{*}{ Tratamiento } & $\mathrm{P}$ & 0.0895 & 0.9224 & 0.1876 \\
\hline & Control & 6.39 & 3.25 & 2.97 \\
\hline & Selenio & 5.90 & 3.10 & 2.60 \\
\hline \multirow{3}{*}{ Tiempo de maduración } & $\mathrm{P}$ & 0.0155 & 0.0079 & 0.5340 \\
\hline & Día 0 & 5.96 & 2.82 & 2.81 \\
\hline & Día 7 & 6.33 & 3.57 & 2.73 \\
\hline \multirow{3}{*}{ Edad } & $\mathrm{P}$ & 0.3856 & 0.0850 & 0.0208 \\
\hline & 20 meses & 6.29 & 2.99 & 2.51 \\
\hline & 36 meses & 6.01 & 3.38 & 3.06 \\
\hline
\end{tabular}

$1=$ escala estructurada del 1 al 9 en forma creciente, siendo 1 nada y 9 muy intenso.

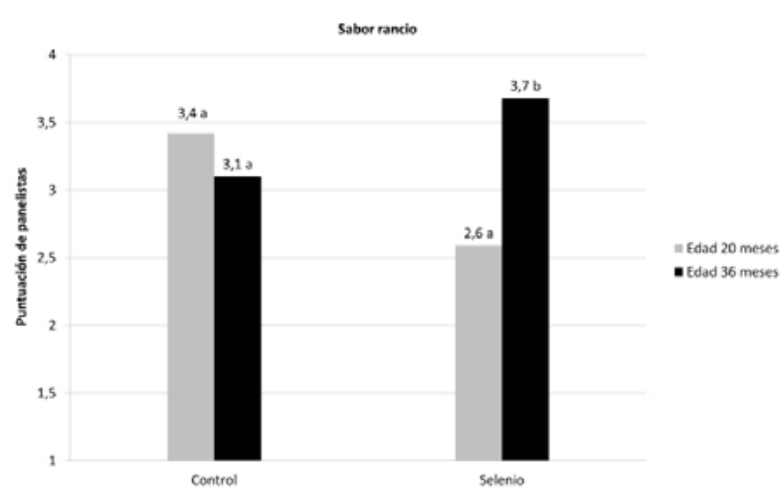

Figura 4: Puntuación del panel sensorial sobre sabor rancio según tratamiento y edad. Escala del 1 (nada) al 9 (muy intenso).

La suplementación con Se tuvo un efecto significativo sobre la oxidación de lípidos, (tabla 3 ) expresado en los menores niveles de MDA, mientras que no se evidenció efecto en la oxidación de proteínas.

Por otra parte, a medida que trascurren los días de exposición al oxígeno en vitrina refrigerada, se observan aumentos de la oxidación tanto de lípidos como de proteínas.

Letras diferentes en la misma columna (A, B, C) difieren estadísticamente. Letras diferentes en la misma fila ( $\mathrm{y}$ y $\mathrm{b}$ ) difieren estadísticamente.

Al analizar la estabilidad del color, vemos que no hubo efecto de la suplementación con selenio (Tabla 4).

En relación a la edad, las vaquillonas de 36 meses mostraron mayores valores de índice de rojo ( $\left.\mathrm{a}^{*}\right)$, índice de amarillo $\left(\mathrm{b}^{*}\right)$ e intensidad de color $\left(\mathrm{C}^{*}\right)$.
Independientemente de la suplementación con Se y la edad del animal, la evolución del color en función de los días de exposición muestran una curva normal, ya que los parámetros $\mathrm{L}^{*}, \mathrm{a}^{*}, \mathrm{~b}^{*}$ y $C^{*}$ aumentan hacia el día 3 y se mantienen hasta el día 6 para luego descender (Figura 3).

La suplementación con selenio no tuvo efecto en los parámetros sensoriales para terneza y sabor extraño (Tabla 5). La carne madurada durante 7 días logró una mayor terneza, aunque el panel detectó una mayor rancidez.

En relación al sabor extraño, ni el tiempo de maduración ni el tratamiento tuvieron efecto. Donde sí se obtuvieron diferencias fue en la edad de las vaquillonas, destacándose la carne de vaquillonas jóvenes las cuales presentaron un menor valor.

Sin embargo, cuando se analizó el efecto de la suplementación con Se, en sabor rancio se detectó una interacción con edad de la vaquillona $(p=0.033)$ que se muestra en la figura 4.

La carne de vaquillonas de 20 meses y que fueron suplementadas con Se, resultaron en un menor sabor rancio que las vaquillonas de 36 meses de edad.

\section{Discusión}

$\mathrm{Al}$ inicio del experimento la actividad de la enzima GSH-Px se encontraba dentro del rango de valores catalogado como carencia marginal (101-130 U/grHb) (Wittwer et al., 2002), mientras que al final del experimento se ubicó dentro de niveles adecuados, por lo que si bien la suplementación con Se tuvo un efecto positivo, los componentes de la dieta también tuvieron influencia en el aumento de la actividad de esta enzima. La mayoría de los trabajos coinciden con estos resultados en evidenciar una mayor respuesta de la enzima GSH-Px en animales suplementados con Se. En este sentido, Juniper et al., (2008), y Lee et al., (2007) reportaron una mayor actividad de esta enzima en los animales que recibían Se orgánico en comparación a los que se les suplementaba con Se inorgánico ( $\mathrm{NaSe})$. 
La baja repercusión del uso de selenio orgánico en la calidad de la canal coinciden con Clyburn (2002), Cozzi et al., (2011), Lee et al., (2007), y Sgoifo Rossi et al., (2015), donde no encontraron diferencias significativas en rendimiento canal y espesor de grasa subcutánea en bovinos. Lawler et al., (2004) mencionan que suplementando con selenio orgánico, a menos que haya una evidente carencia que afecte la tasa de crecimiento, es poco probable que influya en la calidad de la canal.

En cuanto al efecto de la edad del animal en la calidad de la canal, las vaquillonas de 36 meses lograron un mayor rendimiento debido a los mayores pesos de faena y de la canal en relación a las vaquillonas más jóvenes.

En relación a la calidad de la carne y analizando la CRA, la ausencia de respuesta a la suplementación de Se coincide con Clyburn (2002), Sgoifo Rossi et al., (2015), Skřivanová et al., (2007) y Taylor et al., (2008) donde no encontraron diferencias entre fuentes y dosis tanto de Se como vitamina $\mathrm{E}$ en vacunos. Estos resultados podrían estar explicados porque, a diferencia de la GSHPx-1, la enzima GSHPx-4 (que es inducida solamente en dietas deficientes en $\mathrm{Se}$ ) reduce los hidroperóxidos lipídicos y proporciona protección para la integridad y estabilidad de las membranas. En nuestro experimento los niveles iniciales de GSHPx en sangre indicaron una carencia marginal de Se y los animales Control manifestaron respuesta a la GSHPx con la dieta base. Esto probablemente fue suficiente para estimular la capacidad antioxidante de la GSHPx-4 en las membranas celulares lo que explicaría la ausencia de respuesta de los animales suplementados en la CRA (Edens y Sefton, 2016).

Los menores valores de fuerza de corte de las muestras de los animales suplementados con Selenio y madurados durante 7 días en relación a las muestras de 48 horas postmortem coinciden con los resultados de Cozzi et al., (2011) donde encontraron que el tratamiento que fue suplementado con Se orgánico presentó una mayor terneza. De la misma forma, Sgoifo Rossi et al. (2015) reportan una tendencia a una menor fuerza de corte para los animales suplementados con Se orgánico $(p=0,076)$ en comparación con la fuente inorgánica. Esta mejora en la terneza de la carne por efecto del selenio, al igual que Rowe et al. (2004), se atribuye a la preservación de la actividad proteolítica al proteger a la calpaína de la oxidación. Sin embargo, los resultados de nuestro experimento muestran que la suplementación con selenio no tuvo efecto en la oxidación proteica (Tabla 3), por lo que sería necesario profundizar en la investigación para aclarar mejor el papel del Se en el proceso de proteólisis de la carne.

Por otra parte, no se encontraron diferencias significativas en fuerza de corte entre las vaquillonas de 20 y 36 meses, con valores correspondientes a una carne tierna. Shackelford et al., (1995) indicaron que valores de fuerza de corte por encima de $4,5 \mathrm{~kg}$ no son considerados aceptables, y que los grados de satisfacción del consumidor se incrementan cuando esta fuerza es menor a $3,6 \mathrm{~kg}$.
El uso de selenio disminuyó la oxidación lipídica, dando como resultado una menor concentración de los niveles de MDA. Sin embargo la concentración permaneció en niveles de aceptación (< de 1 a 2 mg MDA/kg) (González et al., 2014). Los resultados mencionados en la literatura muestran una baja respuesta antioxidante del selenio y un nivel medio de respuesta cuando está asociado a la vitamina $\mathrm{E}$ en carne de rumiantes (Juniper et al., 2008; O’Grady et al., 2001).

La suplementación con Se no logró disminuir la oxidación proteica ( $p>0,05)$. Una vez que comienzan las reacciones oxidativas, los cambios en la oxidación de los lípidos progresaría más rápido que la degradación oxidativa de las proteínas miofibrilares (Estévez, 2011). Debido a esto, los radicales e hidroperóxidos formados a partir de los lípidos insaturados pueden atacar a las cadenas de aminoácidos susceptibles y producir carbonilos. Los niveles de oxidación lipídica encontrados en este trabajo fueron bajos y quizás no fueron suficientes para desencadenar a posteriori las reacciones de oxidación de proteínas. En la bibliografía no hay reportes de oxidación proteica en los trabajos donde se analiza la suplementación con selenio en vacunos y en ovinos. Los valores encontrados fueron inferiores a los reportados por Terevinto (2010) donde el contenido promedio de carbonilos proteicos hallado en tres músculos de la raza Hereford varió desde 0.89-1.42 nmoles DNPH/mg proteína en estado fresco y desde 1.20-1.41 nmoles DNPH/mg proteína en muestras maduradas.

En relación a la estabilidad del color de la carne, la suplementación con Selenio no tuvo efecto. Estos resultados coinciden con los reportados por Skřivanová et al., (2007) y Taylor et al., (2008), los cuales evaluaron muestras almacenadas en bandejas de polietileno envueltas en film permeable y refrigeradas durante 6 y 12 días respectivamente, no reportando diferencias significativas para ninguno de los parámetros de color analiza$\operatorname{dos}\left(L^{*}, a^{*} y b^{*}\right)(p>0,05)$. Evaluando muestras almacenadas al vacío durante 6 y 11 días a $4{ }^{\circ} \mathrm{C}$ Cozzi et al., (2011) obtuvieron diferencias únicamente en el parámetro de $\mathrm{L}^{*}$ en el día 6 para los tratamientos que contenían Se orgánico en la dieta $(\mathrm{p}<0,01)$. La ausencia de respuesta en las variaciones de color probablemente se deba a que en nuestras condiciones de exposición a un alto contenido de oxígeno, la estabilidad de la oximioglobina se vio favorecida, mientras se desarrollaba la oxidación de lípidos. Esto llevó a que la interacción oxidativa entre los lípidos y la mioglobina no estuviera estrechamente relacionada (Faustman et al., 2010).

Las vaquillonas de 36 meses mostraron mayores valores de índice de rojo (a*), índice de amarillo ( $\left.\mathrm{b}^{*}\right)$ e intensidad de color $\left(C^{*}\right)$ en relación a las de 20 meses. Esto se explica porque los niveles de mioglobina, que es la proteína responsable del color rojo de la carne, aumenta con la edad hasta los 2 años, para luego estabilizarse (Renerre, 1982).

La evolución del color en función de los días de exposición muestran que los parámetros $\mathrm{L}^{*}, \mathrm{a}^{*}, \mathrm{~b}^{*}$ y $\mathrm{C}^{*}$ aumentan hacia el 
día 3 y se mantienen hasta el día 6 para luego descender. El tono $\left(\mathrm{H}^{*}\right)$ aumenta debido a que depende del estado químico de la mioglobina, o sea de la formación de metamioglobina responsable del color amarronado de la carne. Estos datos confirman que la vida útil de la carne en contacto con el oxígeno no supera los 6 días en temperatura de refrigeración.

En la evaluación sensorial, la suplementación con selenio y la edad de las vaquillonas no mostraron un efecto significativo sobre la terneza de la carne, lo cual se corresponde con los valores de fuerza de corte obtenidos en la evaluación instrumental. Las diferencias encontradas en fuerza de corte de $0.300 \mathrm{~kg}$ entre tratamientos y de $0.200 \mathrm{~kg}$ entre las vaquillonas de 20 y 36 meses no fueron detectadas por los panelistas entrenados. Sin embargo, no se identificó la interacción de la evaluación instrumental en donde las vaquillonas suplementadas obtuvieron $0.540 \mathrm{~kg}$ menos en fuerza de corte que las Control, con carne madurada durante 7 días. En 1996 Huffman, citado por Destefanis et al. (2007), encontró que el consumidor puede detectar una diferencia $1 \mathrm{~kg}$ en fuerza de corte realizada por la cizalla de Warner Bratzler, lo cual puede estar explicando estos resultados.

Los panelistas no identificaron diferencias en sabor rancio por efecto de la suplementación con Se a pesar de una disminución en la oxidación de lípidos. Esto puede ser explicado por los bajos valores de MDA obtenidos, incluso en las muestras control. Greene y Cumuze (1981) mencionan que los panelistas son capaces de detectar diferencias en rancidez a partir de valores entre 1 a $2 \mathrm{mg}$ de $\mathrm{MDA} / \mathrm{kg}$ de carne vacuna, de $0.5 \mathrm{mg}$ de $\mathrm{MDA} / \mathrm{kg}$ en carne de cerdo (Dunshea et al., 2005) y de $1 \mathrm{mg}$ de MDA/ $\mathrm{kg}$ en carne de cordero (Ripoll et al., 2011). Sin embargo, los panelistas detectaron un menor sabor rancio en la carne de las vaquillonas más jóvenes y que fueron suplementadas con Se. Este grupo de animales fue el que logró mayor actividad de la GSH-Px en sangre, enzima que proporciona una defensa contra el estrés oxidativo. Además, a medida que aumenta la edad del animal, la composición de ácidos grasos varía, disminuyendo el contenido de ácidos grasos poliinsaturados, debido a que la edad tiende a saturar las grasas, haciéndolas menos susceptibles al daño oxidativo (Montossi y Sañudo, 2007).

Es importante resaltar que no se detectaron muestras con sabor extraño, especialmente sabor metálico, a pesar de los mayores niveles de Se utilizados en este ensayo ( $0.9 \mathrm{ppm})$ en comparación con la mayoría de los trabajos revisados $(0.3 \mathrm{ppm})$, factor que podría ir en desmedro de la aceptación por parte del consumidor.

\section{Conclusiones}

La suplementación de vaquillonas con selenio orgánico en régimen de estabulación logró un aumento de los niveles de la enzima glutatión peroxidasa en sangre, partiendo de niveles de carencia marginal de Se. El tratamiento con Se tuvo efecto en la disminución de la oxidación de los lípidos de la carne lo cual fue detectado por el panel sensorial a través de un menor sabor a rancio en la vaquillonas de 20 meses de edad.

Sin embargo, no hubo efecto sobre los parámetros de calidad de la canal, la estabilización del color durante el almacenamiento y en la oxidación de proteínas de la carne.

Sería necesario profundizar en la investigación para aclarar mejor los mecanismos involucrados sobre el color de la carne, así como el potencial impacto en la oxidación de proteínas.

\section{Agradecimientos}

A la empresa Biotech Uruguay por el aporte del producto Selplex 2000.

Al personal de campo de la Estación Mario A. Cassinoni EEMAC.

\section{Referencias bibliográficas}

Albertí, P., Panea, B., Ripoll, G., Sañudo, C., Olleta, J.L., Hegueruela, I...Serra X. (2005). Medición del color. En V. Cañaque y C. Sañudo (coord.), Estandarización de las metodologías para evaluar la calidad del producto (animal vivo, canal, carne y grasa) en los rumiantes (pp.216-225). Madrid: INIA.

Arthington, J. D. (2008). Effects of supplement type and selenium source on measures of growth and selenium status in yearling beef steers. Journal of Animal Science, 86, 14721477.

Beltrán, J. A. y Roncalés, P. (2000). Determinación de la textura. En V. Cañaque y C. Sañudo (coord.), Metodología para el estudio de la calidad de la canal y de la carne en rumiantes (pp 169-172). Madrid: INIA.

Behrends, J., Mikel, W., Armstrong, C. y Newman, M. (2003). Color stability of Semitendinosus, and Biceps femoris steaks in a high oxygen modified atmosphere. Journal of Animal Science, 81, 2230-2238.

Clyburn, B. S. (2002). Effects of sel-plex (organic selenium) and vitamin $E$ on performance, immune response and beef cut shelf life of feedlot steers. (Tesis doctoral, Texas Tech University). Recuperado de https://tuir.tdl.org/bitstream/ handle $/ 2346 / 8542 / 31295018541473$.pdf? sequence $=1$ \&isAllowed $=\mathrm{y}$

Cozzi, G., Prevedello, P., Stefani, A. L., Piron, A., Contiero, B., Lante, A...Chevaux, E. (2011). Effect of dietary supplementation with different sources of selenium on growth response, selenium blood levels and meat quality of intensively finished Charolais young bulls. Animal, 1531-1538.

Descalzo, A. M., y Sancho, A. M. (2008). A review of natural antioxidants and their effects on oxidative status, odor and quality of fresh beef produced in Argentina. Meat Science 79, 423-436.

Downs, K. M., Hess, J. B. y Bilgili, S. F. (2000). Selenium source effect on broiler carcass characteristics, meat quality and drip loss. Journal of Applied Animal Research, 18, 61-72. 
Destefanis, G., Brugiapaglia, A., Barge, M. T. y Dal Molin, E. (2007). Relationship between beef consumer tenderness perception and Warner-Bratzler shear force. Meat Science 78, 153-156.

Dunshea, F. R., Souza, D. N., Pethick, D. W., Harper, G. S. y Warner, R. D. (2005). Effects of dietary factors and other metabolic modifiers on quality and nutritional value of meat. Meat Science, 71, 8-38.

Edens, F. W. (1996). Organic selenium, from feathers to muscle integrity to drip loss. Five years onward: no more selenite! En Biotechnology in the Feed Industry. Proceedings of Alltech's 12th Annual Symposium (pp 165-185). Nottingham: Nottingham University.

Edens, F.W. y Sefton, A. E. (2016). A role for Sel-Plex ${ }^{\mathrm{TM}}$, a source of organic selenium in salinized yeast cell wall protein, as a factor that influences meat stability. Journal of Applied Animal Nutrition, 4, 11-20.

Estevez, M. (2011). Protein carbonyls in meat systems: A review. Meat Science, 89(3), 259-279.

Faustman, C., Mancini, R., Sun, Q., Surendranath, P. y Suman, P. (2010). Myoglobin and lipid oxidation interactions: Mechanistic bases and control. Meat Science, 86, 86-94.

Geay, Y., Bauchart, D., Hocquette, J. F. y Culioli, J. (2001). Effect of nutritional factors on biochemical, structural and metabolic characteristics of muscles in ruminants, consequences on dietetic value and sensorial of meat. Reproduction Nutrition Development, 41, 1-26.

Greene, B. E. y Cumuze, T. H. (1981). Relationship between TBA numbers and inexperience panellists assessments of oxidized flavour in cooked beef. Journal of Food Science, 47, 52-8.

González, M. I., Mesa, C. A. y Quintero, O. A. (2014). Estimación de la vida útil de almacenamiento de carne de res y de cerdo con diferente contenido graso. Vitae, 21, 201-210.

Immonen, K., Ruusunen, M., Hissa, K. y Puolanne, E. (2000). Bovine muscle glycogen concentration in relation to finishing diet, slaughter and ultimate $\mathrm{pH}$. Meat Science, 55, 25-31.

Juniper, D. T., Phipps, R. H., Ramos-Morales, E. y Bertin, G. (2008). Effect of dietary supplementation with selenium-enriched yeast or sodium selenite on selenium tissue distribution and meat quality in beef cattle. Journal of Animal Science, 86, (11), 3100-3109.

Klont, R. V., Barnier, F., Smulders, A., Van Dijk, A., Hoving-Bolink, C. y Eikelenboom, G. (1999). Post-mortem variation in $\mathrm{pH}$, temperature, and colour profiles of veal carcasses in relation to breed, blood haemoglobin content, and carcass characteristics. Meat Science, 53, 195-202.

Lawler, T. L., Taylor, J. B., Finley, J. W. y Caton, J. S. (2004). Effect of supranutritional and organically bound selenium on performance, carcass characteristics, and selenium distribution in finishing beef steers. Journal of Animal Science, 82, 1488-1493.

Lee, S. H., Park, B. Y., Yeo, J. M., Lee, S. S., Ha, J. K. y Kim, W. Y. (2007). Effects of Different Selenium Sources on Performance, Carcass Characteristics, Plasma Glutathione Peroxidase Activity and Selenium Deposition in Finishing Hanwoo
Steers. Journal of Animal Science, 20(2), 229-236.

Lynch, S. M. y Frei, B. (1993). Mechanisms of copper- and iron-dependent oxidative modification of human low density lipoprotein. Journal of Lipid Research, 34(10), 1745-1751.

Mahan, D. C. y Parrett, N. A. (1996). Evaluating the efficacy of selenium enriched yeast and sodium selenite on tissue selenium retention and serum glutathione peroxidase activity in grower and finisher swine. Journal of Animal Science, 74, 2967-2974.

Masana, M., Meichtri, H. y Rodríguez, H. (2002). Mayor Calidad por Más Tiempo. Revista IDIA XXI, 2(2), 157-162.

Mercier, Y., Gatellier, P. y Renerre, M. (2004). Lipid and protein oxidation in vitro, and antioxidant potential in meat from Charolais cows finished on pasture or mixed diet. Meat Science, 66, 467-473.

Montossi, F. y Sañudo, C. (2007). Cooperación Hispano-Uruguaya: Evolución y promoción de la calidad de la carne bovina y ovina del Uruguay en el mercado europeo. Montevideo: INIA.

Naylor, A. J., Choct, M. y Jacques, K. A. (2000). Effects of selenium source and level on performance and meat quality in male broilers. Poultry Science, 79 (1), 117-121.

O’Grady, M. N., Monahan, F. J., Fallon, R. J. y Allen, P. (2001). Effects of dietary supplementation with vitamin $\mathrm{E}$ and organic selenium on the oxidative stability of beef. Journal of Animal Science, 79, 2827, 2834.

O’Sullivan, A., Galvin, K., Moloney, A. P., Troy, D. J., O’Sullivan, K. y Kerry, J. P. (2003). Effect of pre-slaughter rations of forage and/or concentrates on the compositions and quality of retail packaged beef. Meat Science, 63, 279-286.

Paglia, D. E. y Valentine, W. N. (1967). Studies on the quantitative and qualitative characterization of erythrocyte glutathione peroxidase. Journal of Laboratory and Clinical Medicine, 70(1), 158-69.

Realini, C. E., Duckett, S. K., Brito, G. W., Dalla Rizza, M. y De Mattos, D. (2004). Effect of pasture vs. concentrate feeding with or without antioxidants on carcass characteristics, fatty acid composition, and quality of Uruguayan beef. Meat Science, 66 (3), 567-577.

Renerre, M. (1982). La couleur de la viande et sa mesure. Bulletin Techniche C.R.Z.V., 47-54.

Ripoll, G., Joy, M. y Muñoz, F. (2011). Use of dietary vitamin $\mathrm{E}$ and selenium (Se) to increase the shelf life of modified atmosphere packaged light lamb meat. Meat Science, 87 (1), 88-93.

Rowe, L. J, Maddock, K. R., Lonergan, S. M. y Huff-Lonergan, E. (2004). Oxidative environments decrease tenderization of beef steaks through inactivation of $\mu$-calpain. Journal of Animal Science, 82, 3254-3266.

SAS/stat user's guide release 9.1. (2012). Carey: SAS Institute. Sgoifo Rossi, C. A., Compiani, R., Baldi, G., Bernardi, C. E. M., Muraro, M., Marden, J. P. y Dell'Orto, V. (2015). The effect of different selenium sources during the finishing phase on beef quality. Journal of Animal and Feed Sciences, 24, 93-99.

Shackelford, S. D., Wheeler, T. L. y Koohmaraie, M. (1995). 
Relationship between Shear Force and Trained Sensory Panel Tenderness Ratings of 10 Major Muscles from Bos indicus and Bos taurus Cattle. Journal of Animal Science, 73, 33333340.

Skřivanová, E., Marounek, M., De Smet, S. y Raes, K. (2007). Influence of dietary selenium and vitamin $\mathrm{E}$ on quality of veal. Meat Science, 76, 495-500.

Taylor, J. B., Marchello, M. J., Finley, J. W., Neville, T. L., Combs, G. F. y Caton, J. S. (2008). Nutritive value and display-life attributes of selenium-enriched beef-muscle foods. Journal of Food Composition and Analysis, 21(2), 183-186

Terevinto Herrera, A. (2010). Oxidación lipídica y proteica, capacidad antioxidativa y actividad de las enzimas catalasa, superóxido dismutasa y glutatión peroxidasa en la carne fresca y madurada de novillos Hereford y Braford. (Tesis de Maestría en Ciencias Agrarias, Universidad de la República, Facultad de Agronomía). Recuperado de https://hdl.handle. net/20.500.12008/1815.

Wittwer, F., Araneda, P., Ceballos, A., Contreras, P. A., Andaur, M. y Böhmwald, H. (2002). Actividad de glutatión peroxidasa (GPx1) en sangre de bovinos a pastoreo de la IX Región, Chile y su relación con la concentración de selenio en el forraje. Archivos de Medicina Veterinaria, 34, 49-57.

\section{Notas de contribución:}

1. Concepción y diseño del estudio, 2. Adquisición de datos, 3. Análisis de datos, 4. Discusión de los resultados, 5. Redacción del manuscrito, 6. Aprobación de la versión final del manuscrito.

Juan Franco ha contribuido en 1, 2, 3, 4, 5 y 6. Álvaro Simeone ha contribuido en 1, 2,4 y 6. Virginia Beretta ha contribuido en 1, 3, 4 y 6. Carolina Realini ha contribuido en 1, 3, 4. Ali Saadoun ha contribuido en 2. Alejandra Terevinto ha contribuido en 2. Cristina Cabrera ha contribuido en 2. Agustín Costanzo ha contribuido en 2 y 4, Gonzalo Ravecca ha contribuido en 2 y 4. Antonella Goyeneche ha contribuido en 2 y 4, Rafael Delpiazzo ha contribuido en 2 y 4 . Oscar Betancur ha contribuido en 3.

El editor Cecilia Cajarville aprobó este artículo 